\title{
Clipaje de aneurisma paraclinoideo derecho no roto mediante abordaje endonasal endoscópico: reporte de caso en un centro de tercer nivel en la Ciudad de México
}

Endonasal clipping of unruptured right paraclinoid aneurysm. Case report in a third level center in Mexico City

Mauricio D. Sánchez-Calderón ${ }^{*}$, Diego Ochoa-Cacique, Óscar Medina-Carrillo, Ulises García-González, Antonio Avilés-Aguilar', José R. Aguilar-Calderón ${ }^{1}$, María E. Romero-Cabrera² y León F.I. García-Lara ${ }^{3}$

${ }^{1}$ Departamento de Neurología y Neurocirugía; ${ }^{2}$ Departamento de Anestesiología; ${ }^{3}$ Servicio de Otorrinolaringología y Cirugía de Cabeza y Cuello. Hospital de Alta Especialidad Centro Sur, Petróleos Mexicanos (PEMEX), Ciudad de México, México

\begin{abstract}
Resumen
Los aneurismas paraclinoideos cobran importancia debido a la dificultad técnica por sus relaciones anatómicas, por lo que se han buscado diferentes abordajes para su adecuado manejo. Se presenta el caso de una paciente con un aneurisma paraclinoideo y tratamiento mediante abordaje endonasal con apoyo angiográfico adecuado. Se requieren conocimientos de la anatomía y habilidad quirúrgica para el manejo de estas lesiones, así como asistencia de controles angiográficos para el éxito del tratamiento. El abordaje endonasal endoscópico es adecuado en caso de que la anatomía lo permita y se tenga la infraestructura hospitalaria básica para obtener un alto índice de éxitos.
\end{abstract}

Palabras clave: Aneurisma paraclinoideo no roto. Abordaje endonasal. Clipaje. Incidental.

\begin{abstract}
Paraclinoid aneurysms take importance due to the technical difficulty due to anatomical relationships, which is why different approaches have been sought for their proper management. This is a female patient with a finding of a paraclinoid aneurysm and treatment using an endonasal approach with adequate angiographic support. Knowledge of the anatomy and surgical skill are required for the management of these lesions, assisted by angiographic controls for successful treatment. The endoscopic endonasal approach is adequate if the anatomy allows it and the basic hospital infrastructure is available to obtain high success rates.
\end{abstract}

Key words: Unruptured paraclinoid aneurysm. Endonasal approach. Clipping. Incidental.

\section{Correspondencia:}

*Mauricio D. Sánchez-Calderón

Avda. La Hacienda 126

Col. Residencial Villa Coapa, Del. Tlalpan

C.P. 14390 , Ciudad de México, México

Fecha de recepción: 28-01-2021

Fecha de aceptación: 01-03-2021

Cir Cir. 2021;89(S2):38-40

E-mail: md.sanchez.neurocirugia@gmail.com

DOI: $10.24875 / \mathrm{CIRU} .21000071$

Contents available at PubMed

www.cirugiaycirujanos.com

0009-7411/@ 2021 Academia Mexicana de Cirugía. Publicado por Permanyer. Este es un artículo open access bajo la licencia CC BY-NC-ND (http://creativecommons.org/licenses/by-nc-nd/4.0/). 


\section{Introducción}

La enfermedad cerebral aneurismática tiene especial importancia en la morbimortalidad internacional. La incidencia mundial es de aproximadamente el $6 \%$, siendo mayor en población asiática. De los pacientes que sufren una rotura aneurismática, aproximadamente el 30\% fallecerán, el 30\% tendrán secuelas neurológicas importantes y el 30\% serán socialmente funcionales. Los aneurismas paraclinoideos ocupan un importante lugar debido a la dificultad técnica, por lo cual se han buscado opciones terapéuticas, entre ellas el manejo endovascular y el abordaje transnasal endoscópico'; este último solo se recomienda en casos bien seleccionados y que por medio de otras técnicas tengan un mayor riesgo de complicaciones.

\section{Caso clínico}

Mujer de 43 años con cefalea progresiva de 2 semanas de evolución asociada a galactorrea y unos valores de prolactina de $32.5 \mathrm{ng} / \mathrm{ml}$. En la exploración física no se observan datos de focalidad neurológica. Se realizó una angiotomografía (Fig. 1) y por los hallazgos se realizó una angiografía cerebral, que fue diagnóstica (Fig. 2). Se programó para clipaje de aneurisma con abordaje endonasal endoscópico y se utilizó un clip recto de $7 \mathrm{~mm}$ (Fig. 3) con controles angiográficos transquirúrgicos (Fig. 4), así como control proximal con balón a nivel de la arteria carótida petrosa. Presentó una elevación transquirúrgica de los volúmenes urinarios con respuesta adecuada a la desmopresina. Se realizó control angiográfico posquirúrgico que mostró la exclusión total de la lesión vascular (Fig. 5). La paciente continuó con una evolución favorable y sin complicaciones posquirúrgicas.

\section{Discusión}

Con el auge de los procedimientos endovasculares se han buscado opciones alternativas para las lesiones vasculares de díficil acceso ${ }^{2}$. El clipaje endonasal requiere amplios conocimientos anatómicos y habilidades quirúrgicas excepcionales por la dificultad del control proximal que se requiere para ofrecer una cirugía segura. Se considera este abordaje como mínima invasión y su ventaja con respecto a otros abordajes es la exposición total del domo del aneurisma, así como de su cuello y, sobre todo, las arterias perforantes adyacentes; además, permite la manipulación

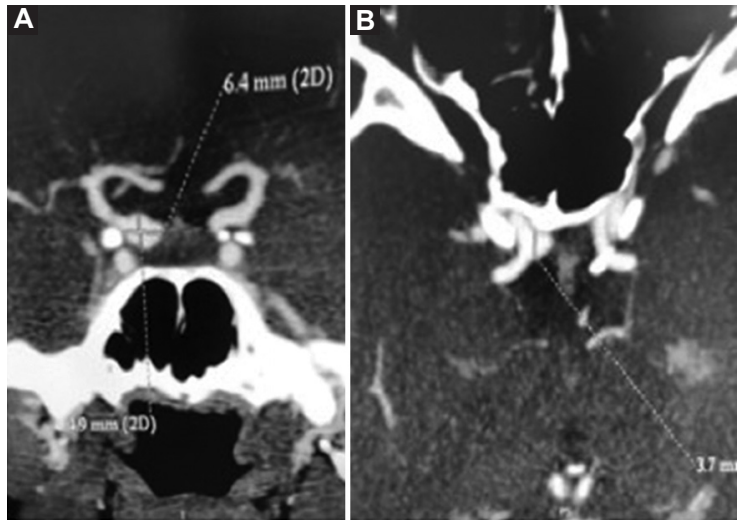

Figura 1. Angiotomografía de cráneo. A: corte coronal. B: corte axial. Se observa una imagen sacular con dimensiones de $6.4 \times 4.9 \mathrm{~mm}$ en el segmento paraclinoideo de la arteria carótida interna derecha.

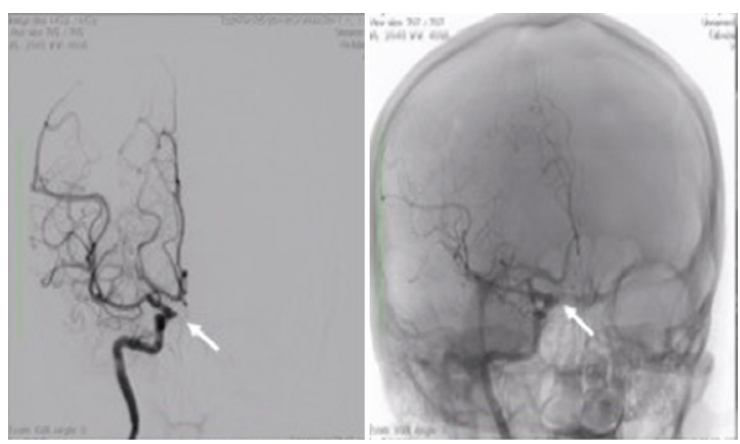

Figura 2. Angiografía preoperatoria en proyección anteroposterior y reconstrucción de $360^{\circ}$, que muestra una lesión vascular (flecha) en el segmento paraclinoideo.

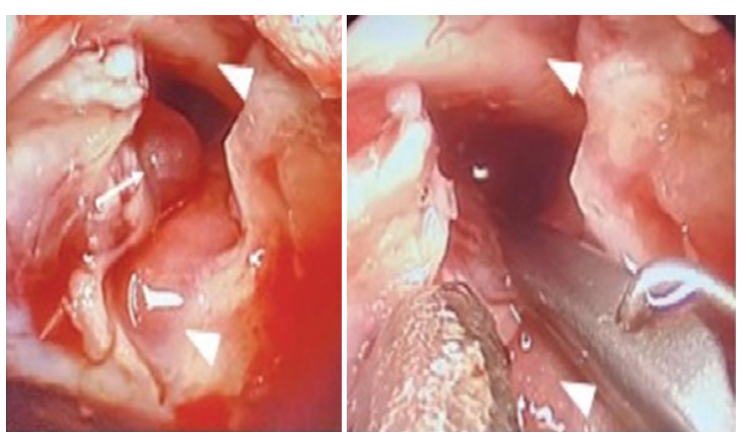

Figura 3. Vista endoscópica que muestra la lesión vascular en el centro de la imagen y el quiasma óptico en la parte superior, así como la posición del clip y la exclusión del aneurisma.

cerebral transquirúrgica y una recuperación clínica más «cómoda» ${ }^{3}$.

Los aneurismas paraclinoideos cobran importancia quirúrgica debido a su complejidad técnica y difícil 


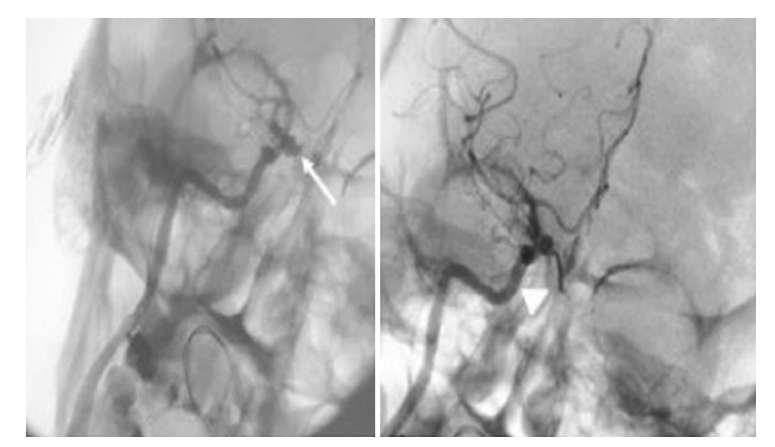

Figura 4. Angiografía transoperatoria en proyección anteroposterior que muestra la lesión vascular y la posición del clip, así como la exclusión de la lesión.

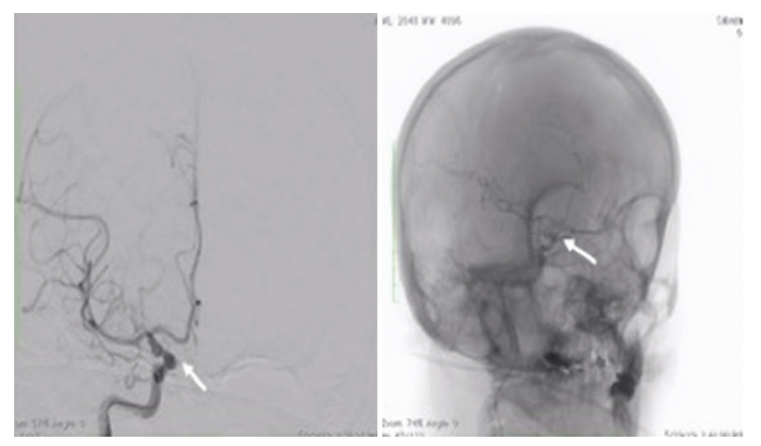

Figura 5. Angiografía posoperatoria en proyección anteroposterior y reconstrucción de $360^{\circ}$ que muestra la posición del clip (flecha) y la exclusión de la lesión aneurismática.

acceso por abordaje abierto ${ }^{4}$. En los aneurismas localizados en segmentos paraclinoideos (oftálmicos inferiores, hipofisarios y segmento cavernoso) y cuando la disposición del domo sea medial puede ser mejor opción el abordaje endonasal, al contrario que en los aneurismas en la circulación posterior, que pueden presentar mayor dificultad quirúrgica ${ }^{5}$.

\section{Conclusiones}

La anatomía de las arterias hipofisarias superiores es compleja y su intervención se favorece con la visualización directa asistida por abordajes endoscópicos endonasales para una adecuada exposición de la zona periquiasmática, y es primordial el control vascular para mantener la adecuada perfusión de los tejidos ${ }^{6}$. El abordaje endonasal para el manejo de los aneurismas paraclinoideos es una opción adecuada en caso de lesiones que por otras vías de acceso sean difíciles de manipular y se incremente el riesgo de rotura transquirúrgica; sin embargo, es necesario un entrenamiento especializado para ofrecer un manejo quirúrgico seguro.

\section{Agradecimientos}

A nuestros maestros y compañeros que hicieron posible la realización de este abordaje quirúrgico.

\section{Responsabilidades éticas}

Protección de personas y animales. Los autores declaran que para esta investigación no se han realizado experimentos en seres humanos ni en animales.

Confidencialidad de los datos. Los autores declaran que han seguido los protocolos de su centro de trabajo sobre la publicación de datos de pacientes.

Derecho a la privacidad y consentimiento informado. Los autores han obtenido el consentimiento informado de los pacientes y/o sujetos referidos en el artículo. Este documento obra en poder del autor de correspondencia.

\section{Conflicto de intereses}

Este trabajo no originó ningún conflicto de intereses.

\section{Financiamiento}

Los autores declaran que no se requirió de financiamiento externo para la realización de este manuscrito.

\section{Bibliografía}

1. Tayebi Meybodi A, Borba Moreira L, Little AS, Lawton MT, Preul MC. Anatomical assessment of the endoscopic endonasal approach for the treatment of paraclinoid aneurysms. J Neurosurg. 2018;131:1734-42.

2. Szentirmai O, Hong Y, Mascarenhas L, Salek AA, Stieg PE, Anand VK, et al. Endoscopic endonasal clip ligation of cerebral aneurysms: an anatomical feasibility study and future directions. J Neurosurg. 2016; 124:463-8.

3. Heiferman DM, Somasundaram A, Alvarado AJ, Zanation AM, Pittman AL, Germanwala AV. The endonasal approach for treatment of cerebral aneurysms: a critical review of the literature. Clin Neurol Neurosurg. 2015;134:91-7.

4. Javalkar V, Banerjee AD, Nanda A. Paraclinoid carotid aneurysms. J Clin Neurosci. 2011;18:13-22.

5. Kassam AB, Gardner PA, Mintz A, Snyderman CH, Carrau RL, Horowitz M. Endoscopic endonasal clipping of an unsecured superior hypophyseal artery aneurysm. J Neurosurg. 2007;107:1047-52.

6. Doglietto F, Prevedello DM, Belotti F, Ferrari M, Lancini D, Schreiber A, et al. The superior hypophyseal arteries: anatomical study with an endoscopic endonasal perspective. Oper Neurosurg (Hagerstown). 2019;17:321-31. 